\title{
Quantization of the Jackiw-Teitelboim model in the Temporal Gauge: Quantum dynamics
}

\author{
José André Lourenço \\ Departamento de Ciências Naturais, Universidade Federal do Espírito Santo, São Mateus, ES, Brazil \\ jose.lourenco@ufes.br
}

\begin{abstract}
In this work we study the Jackiw-Teitelboim model (JT model), as a model that has the structure of a BF-type topological theory and we treat the dynamics of the model at the quantum level through the implementation of the constraints originating from the classical theory of the JT model in the temporal gauge in an appropriate Hilbert space.
\end{abstract}

\section{Introduction}

In two dimensional space-time, gravitation can be seen as a gauge theory characterized by the Poincaré group $I S O(1,1)$. As this group does not admit an invariant and nondegenerate quadratic form, the JT model is based on the (Anti)-de Sitter group "(A)dS", the group $S O(2,1)$, which contains the Lorentz group as a subgroup and corresponds to a gravitation theory with a cosmological constant. We see then that the (A)dS group, taken as a gauge group, contains naturally the diffeomorphism symmetry. In this line we investigate the canonical formulation of the JT model [1,2] in order to quantize it through the Loop Quantum Gravity (LQG) formalism $[4,5]$. Following Dirac's program of canonical quantization applied to the loop formalism, we obtain a quantum configuration space. We build the respective kinematic Hilbert space and we define the volume operator in a consistent way. Finally, we treat the dynamics of the model at the quantum level through the implementation of the constraints originating from the classical theory of the JT model in the temporal gauge in an appropriate Hilbert space.

\section{The Jackiw-Teitelboim Model}

\subsection{The Jackiw-Teitelboim action}

Pure Gravity in 2 space-time dimensions cannot be based on the Einstein-Hilbert action $\int d^{2} x \sqrt{-g} R$, which is a surface integral, corresponding to an identically vanishing Einstein tensor $R_{\mu \nu}-\frac{1}{2} g_{\mu \nu} R \equiv$ 0 . A simple but nontrivial model has been proposed long ago independently by Jackiw and by Teitelboim, $R-2 \Omega=0$, as a replacement for the 2-dimensional Ein- 


\section{Blucher Proceedings \\ VII Encontro Científico de Física Aplicada Blucher}

stein equation, which may be derived from the action $[1,2]$,

$$
S_{J T}=\frac{1}{2} \int d^{2} x \sqrt{-g} \psi(R-2 \Omega),
$$

where, the scalar field $\psi$ acts like a Lagrange multiplier.

\subsection{BF Model}

The action $S_{J T}$ can be obtained from a BF type theory, for details see [1, 2]. In this formulation of gravitation in 2 dimensions, we have a gauge connection 1-form A,

$$
A(x)=e^{I}(x) P_{I}+\omega(x) \Lambda,
$$

where $P_{I}(I=0,1)$ are space-time translations and $\Lambda$ is Lorentz boost.

The coefficients in (2) are the zweibein and Lorentz connection forms

$$
e^{I}=e_{\mu}^{I} d x^{\mu}, \quad \omega=\omega_{\mu} d x^{\mu} .
$$

The $B F$ action $S_{B F}$ reads

$S_{B F}[A, \phi]=\int d t d x\left(\partial_{t} A_{x}^{i} \phi_{i}+A_{t}^{i} D_{x} \phi_{i}\right)$,

where $D_{x}$ is a covariant derivative and $\left(\phi_{i}\right)=:\left(\varphi_{0}, \varphi_{1}, \psi\right)$.

The action (4), which is invariant under the (A)dS gauge transformations, turns out to be automatically invariant under the diffeomorphisms, on shell, as a general result for topological theories of this is type. A Legendre transformation yields the Hamiltonian

$$
H=-\int d x A_{t}^{i} D_{x} \phi_{i}
$$

The Poisson bracket algebra is defined by the brackets of the generalized coordinates and their conjugate momenta. For details see $[1,2]$.

\footnotetext{
${ }^{1}$ The vector field $\varepsilon^{i}$ is an arbitrary test function.
}

\subsection{Constraints Algebra in the Temporal Gauge}

Following an approach commonly used for the 4-dimensional theory, as described e.g. in the review [4], we introduce a parcial gauge fixing, the "temporal gauge", which consists in making vanish the component $\chi:=e_{x}^{0}$ of the zweibein $[1,2]$. This condition is implemented as a constraint, $\chi \approx 0$.

With this condition, we have two first class constraints,

$$
\begin{aligned}
\mathscr{G}_{0} & =\partial_{x}^{2} \psi(x) \frac{\sigma}{e_{x}^{1}(x)} \\
& -\partial_{x} \psi(x) \partial_{x} e_{x}^{1}(x) \frac{\sigma}{\left(e_{x}^{1}(x)\right)^{2}} \\
& +\Omega \psi(x) e_{x}^{1}(x)-\varphi_{1}(x) \omega(x),(5) \\
\mathscr{G}_{1} & =\partial_{x} \varphi_{1}+\omega_{x} \partial_{x} \psi .
\end{aligned}
$$

The Dirac bracket algebra of these constraints is given by

$$
\begin{aligned}
\left\{\mathscr{G}_{0}(\varepsilon), \mathscr{G}_{0}(\eta)\right\}_{D} & =\sigma \mathscr{G}_{1}\left(\frac{1}{e}[\varepsilon, \eta]\right), \\
\left\{\mathscr{G}_{0}(\varepsilon), \mathscr{G}_{1}(\eta)\right\}_{D} & =\mathscr{G}_{0}\left(\frac{1}{e} \eta \partial \varepsilon\right), \\
\left\{\mathscr{G}_{1}(\varepsilon), \mathscr{G}_{1}(\eta)\right\}_{D} & =-\mathscr{G}_{1}\left(\frac{1}{e}[\varepsilon, \eta]\right),
\end{aligned}
$$

where $\mathscr{G}_{i}(\varepsilon)=\int d x \varepsilon^{i}(x) \mathscr{G}_{i}(x)^{1}$ and $[\varepsilon, \eta]=\left(\varepsilon \partial_{x} \eta-\eta \partial_{x} \varepsilon\right)$. For details see $[1,2]$.

The independent dynamical variables are the fields $e_{x}^{1}, \omega_{x}, \varphi_{1}$ and $\psi$. Their nonvanishing Dirac brackets are

$$
\left\{e_{x}^{1}(x), \varphi_{1}(y)\right\}_{D}=\{\omega(x), \psi(y)\}_{D}=\delta(x-y) .
$$

The final Hamiltonian is

$H_{F}=-\int d x\left(\mathscr{N}^{0}(x) \mathscr{G}_{0}(x)+\mathscr{N}^{1}(x) \mathscr{G}_{1}(x)\right)$,

where $\mathscr{N}^{0}(x)$ and $\mathscr{N}^{1}(x)$ are arbitrary functions. 


\section{Quantization of the JT Model}

\subsection{Quantization}

Given the fields $e_{x}^{1}(x), \omega(x)$ and the conjugate momenta $\varphi_{1}(x), \psi(x)$, choose the representation $\Psi\left[\varphi_{1}, \psi\right]$ with

$\hat{e}_{x}^{1}(x)=i \hbar \frac{\delta}{\delta \varphi_{1}(x)}, \quad \hat{\omega}_{x}(x)=i \hbar \frac{\delta}{\delta \psi(x)}$.

The quantum algebra of elementary variables is generated by point holonomy at each point of $\mathscr{M}_{1} \cong \mathbb{R}$,

$$
\begin{aligned}
& h_{\lambda}(x):=\exp \left[-i \lambda \varphi_{1}(x)\right], \\
& g_{\mu}(y):=\exp [-i \mu \psi(y)] .
\end{aligned}
$$

The smeared momenta on a 1-dimensional region $I \subset \mathscr{M}_{1}$ are expressed by

$\hat{e}(I):=\int_{I} d x \hat{e}_{x}^{1}(x), \hat{\omega}(I):=\int_{I} d x \hat{\omega}_{x}(x) .(10)$

For details see $[2,3]$.

\subsection{Graphs and spin-networks}

In the case of the field $\varphi_{1}(x)$, a graph is given by $X=\left\{x_{1}, \ldots, x_{n}\right\}$ in which each point $x_{i}$ is called the "edge". To each edge of $X$ it is associated a real arbitrary, nonzero number, $\lambda_{i}$, and the graph $X$ is said to be a colored graph or spin-network, $X(\vec{\lambda}$ ), where $\vec{\lambda} \equiv\left(\lambda_{1}, \ldots, \lambda_{n}\right)$. In the case of the field $\psi(x)$, a graph is given by $Y(\vec{\mu})$, where $\vec{\mu} \equiv\left(\mu_{1}, \ldots, \mu_{m}\right)$ is the so called scalar-network. For details see [2, 3].

\subsection{Cylindrical Functions}

Given the colored graph $\Gamma(\vec{\lambda}, \vec{\mu}) \equiv X(\vec{\lambda}$ )$\cup Y(\vec{\mu})$, then $\mathrm{Cyl}_{\Gamma(\vec{\lambda}, \vec{\mu})}$ is the vector

\footnotetext{
${ }^{2} \mathscr{H}_{\text {Kin }}$ is a non-separable Hilbert space.
}

space generated by finite linear combinations of the following functions

$\mathscr{N}_{\Gamma(\vec{\lambda}, \vec{\mu})}\left(\varphi_{1}, \psi\right)=\mathscr{N}_{X(\vec{\lambda})}\left(\varphi_{1}\right) \otimes \mathscr{N}_{Y(\vec{\mu})}(\psi)$,

where,

$$
\begin{aligned}
& \mathscr{N}_{X(\vec{\lambda})}\left(\varphi_{1}\right)=\prod_{x_{j} \in X} \exp \left[-i \lambda_{j} \varphi_{1}\left(x_{j}\right)\right] \\
& \mathscr{N}_{Y(\vec{\mu})}(\psi)=\prod_{y_{k} \in Y} \exp \left[-i \mu_{k} \psi\left(x_{k}\right)\right] .
\end{aligned}
$$

The space Cyl of all cylindrical functions is defined by

$$
\mathrm{Cyl}=\bigcup_{\Gamma(\vec{\lambda}, \vec{\mu})} \mathrm{Cyl}_{\Gamma(\vec{\lambda}, \vec{\mu})} .
$$

For details see $[2,3]$.

\subsection{Scalar Product}

The scalar product of Cyl is defined by

$$
<\mathscr{N}_{\Gamma(\vec{\lambda}, \vec{\mu})}\left|\mathscr{N}_{\Gamma^{\prime}\left(\overrightarrow{\lambda^{\prime}}, \overrightarrow{\mu^{\prime}}\right)}\right\rangle=\delta_{\Gamma \Gamma^{\prime}} \delta_{\vec{\lambda} \lambda^{\prime}} \delta_{\vec{\mu}} \rightarrow
$$

The total kinematical Hilbert space $\mathscr{H}_{\text {Kin }}$ is the Cauchy completion of the space of cylindrical functions Cyl in the norm defined by the scalar product ${ }^{2}$. For details see $[2,3,4,5]$.

\subsection{Operators}

We can represent the cylindrical functions $\mathscr{N}_{\Gamma(\vec{\lambda}, \vec{\mu})}\left(\varphi_{1}, \psi\right)$ by kets

$$
|\Gamma, \vec{\lambda}, \vec{\mu}>=| X, \vec{\lambda}>\otimes \mid Y, \vec{\mu}_{>} .
$$

The configuration operators (9), act on any cylindrical function as

$$
\begin{aligned}
& \hat{h}_{\lambda_{0}}|\Gamma, \lambda, \mu>=| \Gamma, \lambda+\lambda_{0}, \mu>, \\
& \hat{g}_{\mu_{0}}|\Gamma, \lambda, \mu>=| \Gamma, \lambda, \mu+\mu_{0}>,
\end{aligned}
$$




\section{Blucher Proceedings \\ VII Encontro Científico de Física Aplicada Blucher}

where $X=\left\{x_{0}\right\}, Y=\left\{y_{0}\right\}$. All these operators are unitary.

The momentum operator $\hat{e}(I)$ (10) acts as

$\hat{e}(I)\left|\Gamma, \vec{\lambda}, \vec{\mu}>=\left[\hbar \sum_{x_{j} \in X} \lambda_{j} \chi_{I}\left(x_{j}\right)\right]\right| \Gamma, \vec{\lambda}, \vec{\mu}>$

Its eigenvalues are thus given by $\lambda_{I} \equiv$ $\hbar \sum_{x_{j} \in X} \lambda_{j} \chi_{I}\left(x_{j}\right)$, where the present $\lambda_{j}$ are the continuous analogs of fluxons or spins of quantum geometry. The action of the operator $\hat{\omega}$ can be write in an analogous way. For details see [2, 3].

\subsection{Volume Operator}

The operator corresponding to the "volume" $V(I)$ of a spatial region $I \subset \mathscr{M}_{1}$ acting on scalar-spin-network states, is defined as

$$
\begin{aligned}
& \hat{V}(I) \mid \Gamma, \vec{\lambda}, \vec{\mu}>=\left[\hbar \sum_{x_{j} \in \mathscr{E}(X \cap I)}\left|\lambda_{j}\right|\right] \\
& \text {. } \mid \Gamma, \vec{\lambda}, \vec{\mu}>\text {. }
\end{aligned}
$$

This expression shows that the scalarspin-networks in $\mathscr{H}_{\text {Kin }}$ diagonalize the operator $\hat{V}(I), \forall I$, with eigenvalues given by finite sums, where $\lambda_{j}$ are arbitrary real numbers and the spectrum of the volume operator, denoted by $\sigma(\hat{V})$, is discrete in the sense that we have a base ortonormal (uncountable) that diagonalizes the operator $\hat{V}(I)$. For details see [2, 3].

\section{Implementation of the operators to the quan- tum level}

\subsection{Quantum Dynamics}

- The scalar constraint $\mathscr{G}_{0}(\varepsilon)$ is tremendously non-linear.
- $\left\{\mathscr{G}_{0}(\varepsilon), \mathscr{G}_{0}(\eta)\right\}_{\mathrm{D}}=\sigma \mathscr{G}_{1}\left(\frac{1}{e}[\varepsilon, \eta]\right)$ is not a Lie algebra due to the structure functions.

These facts imply that the operator cannot be defined directly on $\mathscr{H}_{\text {Kin }}$ consistently.

What is the idea to circumvent this problem?

\subsection{Regularization of the scalar constraint $\mathscr{G}_{0}$}

The idea is to build the smeared version of the classical scalar constraint $\mathscr{G}_{0}(x)(5)$,

$$
\begin{aligned}
\mathscr{G}_{0}(N) & =\int_{\mathscr{M}_{1}} d x N(x)\left(\partial_{x}^{2} \psi(x) \frac{\sigma}{e_{x}^{1}(x)}\right. \\
& -\partial_{x} \psi(x) \partial_{x} e_{x}^{1}(x) \frac{\sigma}{\left(e_{x}^{1}(x)\right)^{2}}+\Omega \psi(x) e_{x}^{1}(x) \\
& \left.-\varphi_{1}(x) \omega(x)\right) .
\end{aligned}
$$

We can write $\mathscr{G}_{0}$ by means of Poisson Brackets among quantities that are simple enough to consider their quantization on $\mathscr{H}_{\text {Kin }}$. Now, in order to obtain a regularized version of $\mathscr{G}_{0}$, use the notion of segmentation. In our context, a segmentation $S$ is a decomposition of the real line, so that, $\mathbb{R}=\cup_{I_{l} \in S} I_{l}$, where $I_{l}=\left[L_{l}, R_{l}\right)$.

The regularized version of $\mathscr{G}_{0}$ with respect to a give segmentation $S(\varepsilon)$ of the $\mathscr{M}_{1}$ is

$$
\begin{aligned}
& \mathscr{G}_{0}(N)=\lim _{\varepsilon \rightarrow 0} \sum_{l} N_{l} \\
\cdot & {\left[\frac{i \sigma}{\beta}\left(g_{L_{l}}^{-1}\left(g_{L_{l+2}}-2 g_{L_{l+1}}+g_{L_{l}}\right)+\cdots\right)\right.} \\
\cdot & \left(\frac{2 i}{\alpha} h_{L_{l}}^{-1}\left\{V_{I_{l}}^{\frac{1}{2}}, h_{L_{l}}\right\}_{\mathrm{D}}\right)^{3} V_{I_{l}}^{\frac{1}{2}}+\frac{2 \sigma}{\alpha \beta} U_{L_{l}}^{-1}\left(U_{L_{l+1}}-U_{L_{l}}\right) \\
\cdot & \left(h_{L_{l+1}}^{-1}\left\{V_{I_{l+1}}^{\frac{1}{2}}, h_{L_{l+1}}\right\}_{\mathrm{D}} V_{I_{l+1}}^{\frac{3}{2}}-h_{L_{l}}^{-1}\left\{V_{I_{l}}^{\frac{1}{2}}, h_{L_{l}}\right\} V_{\mathrm{D}}^{\frac{3}{2}}\right) \\
\cdot & \left(\frac{2 i}{\alpha} h_{L_{l}}^{-1}\left\{V_{I_{l}}^{\frac{1}{2}}, h_{L_{l}}\right\}_{\mathrm{D}}\right)^{6} V_{I_{l}}-\frac{\Omega}{\alpha \beta}\left(g_{L_{l}}-g_{L_{l}}^{-1}\right) \\
\cdot & \left.h_{L_{l}}^{-1}\left\{V_{I_{l}}^{\frac{1}{2}}, h_{L_{l}}\right\}_{\mathrm{D}} V_{I_{l}}^{\frac{3}{2}}-\frac{i}{2 \alpha}\left(h_{L_{l}}-h_{L_{l}}^{-1}\right) \omega_{I_{l}}\right],
\end{aligned}
$$




\section{Blucher Proceedings VII Encontro Científico de Física Aplicada Blucher}

where $\alpha \varphi_{1}, \beta \psi<<1$ for all point $L_{l}$. The task now, is to promote the regularized expression (12) to an operator. In our case the segmentation $\mathrm{S}(\varepsilon)$, has a simple property: The graph $\Gamma(\vec{\lambda}, \vec{\mu})$ is embedded in $S(\varepsilon)$ for all $\varepsilon$, so that every edge of $\Gamma$ (in our case the points $x_{k}$ ) coincides with a vertex $L_{l}$ in $S(\varepsilon)$. Then each interval $I_{l}$ of the segmentation $S(\varepsilon)$ contains at most one edge of the $\Gamma$. For details see $[2,3,5]$. The quantum constraint $\widehat{\mathscr{G}}_{0}(N)$, can formally written as

$$
\begin{aligned}
& \widehat{\mathscr{G}}_{0}(N)=\frac{8 i \sigma}{\alpha_{3} \beta \hbar^{3}} \lim _{\varepsilon \rightarrow 0} \sum_{k} N_{k} \\
\cdot & {\left[\left(\hat{g}_{L_{k}}^{-1}\left(\hat{g}_{L_{k+2}}-2 \hat{g}_{L_{k+1}}+\hat{g}_{L_{k}}\right)+\cdots\right)\right.} \\
\cdot & \hat{M}_{L_{k}}^{3} \hat{V}_{I_{k}}^{\frac{1}{2}}-\frac{16}{\alpha^{4} \hbar^{\hbar^{2}}} \hat{g}_{L_{k}}^{-1}\left(\hat{g}_{L_{k+1}}-\hat{g}_{L_{k}}\right) \\
\cdot & \left(\hat{M}_{L_{k+1}} \hat{V}_{I_{k+1}}^{\frac{3}{2}}-\hat{M}_{L_{k}} \hat{V}_{I_{k}}^{\frac{3}{2}}\right) \cdot \hat{M}_{L_{k}}^{6} \hat{V}_{I_{k}} \\
+ & \frac{\Omega \alpha^{2} \hbar^{2} \sigma}{8}\left(\hat{g}_{L_{k}}-\hat{g}_{L_{k}}^{-1}\right) \cdot \hat{M}_{L_{k}} \hat{V}_{I_{k}}^{\frac{3}{2}} \\
- & \left.\frac{\alpha^{2} \beta \sigma \hbar^{3}}{16}\left(\hat{h}_{L_{k}}-\hat{h}_{L_{k}}^{-1}\right) \hat{\omega}_{I_{k}}\right],
\end{aligned}
$$

where, $\hat{M}_{L_{k}}:=\hat{h}_{L_{k}}^{-1}\left[\hat{V}_{I_{k}}^{\frac{1}{2}}, \hat{h}_{L_{k}}\right]$.

Now we analyze the removal of the regulator. Since the only dependence of $\varepsilon$ is in the position of the extra "edge" in the resulting scalar-spin-network states ${ }^{3}$, the limit $\varepsilon \rightarrow 0$ can be defined in the Hilbert space of diffeomorphism invariant states $\mathscr{H}_{\text {Diff. }}$. The key property is that in the context of diffeomorphism invariance the position of the new edges is irrelevant. For details see $[2,3,5]$.

In $\mathscr{H}_{\text {Diff }}$, we have

$$
\left(\Upsilon(\varphi)\left|\left[\widehat{\mathscr{G}}_{0}(N), \widehat{\mathscr{G}_{0}}(M)\right]\right| \psi_{\Gamma}>=0,\right.
$$

for all $\left(\Upsilon(\varphi) \mid \in \mathscr{H}_{\text {Diff }} \subset \mathrm{Cyl}^{*}\right.$ and for all $\mid \psi_{\Gamma}>\in \mathscr{H}_{\text {Kin }}$.
The expression (14) is consistent with the classical expression in which two scalar constraint operators commute on diffeomorphism invariant states. For details see $[2,3,5]$.

We conclude this section by defining the physical Hilbert space $\mathscr{H}_{\text {Phys }} \subset \mathscr{H}_{\text {Diff }}$ as the space of states $(\Upsilon(\varphi) \mid$ so that,

$$
\left(\Upsilon(\varphi)\left|\widehat{\mathscr{G}}_{0}(N)\right| \psi_{\Gamma}>=0,\right.
$$

where one of the possible solutions can be given in terms of

$$
\left|\psi_{\Gamma}>=\sum c(\Gamma, \vec{\mu})\right| \Gamma, \overrightarrow{0}, \vec{\mu}>.
$$

\section{References}

[1] C. P. Constantinidis, J. A. Lourenço, I. Morales, O. Piguet and A. Rios, "Canonical analysis of the JackiwTeitelboim model in the temporal gauge: I. The classical theory", Class. Quantum Grav. 25 (2008) 125003.

[2] J. A. Lourenço, $\mathrm{PhD}$ thesis: "Quantização do Modelo de JackiwTeitelboim no Gauge Temporal via o formalismo de laços", UFES Brazil, 2009.

[3] J. A. Lourenço (work in progress).

[4] A. Ashtekar and J. Lewandowski, "Background independent quantum gravity: A Status report", Class. Quantum Grav. 21 (2004) R53.

[5] T. Thiemann,"Modern Canonical Quantum General Relativity", Cambridge Monographs on Mathematical Physics, 2007.

\footnotetext{
${ }^{3}$ The scalar constraint modifies scalar-spin-networks by creating new "edges".
} 